\title{
Status Report on HIV Drug Treatment Transition from Tenofovir/Lamivudine/Efavirenz (TLE 600mg) to
}

\section{Tenofovir/Lamivudine/Dolutegravir (TLD) in Malawi}

\author{
Babaye $\mathrm{Y}^{1 *}$ and Nyirenda $\mathrm{RK}^{2}$ \\ 1University of Washington, USA \\ ${ }^{2}$ Department of HIV/AIDS, Ministry of Health, Malawi
}

*Corresponding author: Yusuf Babaye, University of Washington, USA, Tel: +2348037878013; E-mail: yusufbabaye2@gmail.com

\section{Editorial}

Volume 2 Issue 1

Received Date: February 16, 2018

Published Date: February 22, 2018

DOI: $10.23880 /$ phoa-16000118
Abbreviations: ART: Anti Retroviral therapy; MOH: Ministry of Health; PLHIV: People Living with HIV; DTG: Dolutegravir; INSTI: Integrase Inhibitor; EFV: Efavirenz; PPM: Pooled Procurement Mechanism; CSOs: Civil Society Organizations; NRTI: Nucleoside Reverse Transcriptase Inhibitors.

\section{Background}

The Malawi Antiretroviral therapy (ART) program has over 740,000 patients on ART by end of September, 2017. This represents $71 \%$ of the estimated 1,036,000 HIV positive population [1]. In December 2017, the Ministry of Health $(\mathrm{MoH})$ in Malawi made a decision to adopt a new drug combination which is Tenofovir/ Lamivudine/ Dolutegravir (TLD) as preferred first-line regimen for ART. In order to ensure adequate preparations the migration to TLD regimen is incorporated in to Malawi 2018 ART/PMTCT guideline review process with overarching goal of providing evidence-based guidance to health workers who support care and treatment services to people living with HIV (PLHIV). Pilot implementation is planned for 3000-5000 patients in the second half of 2018.

\section{Key Considerations for Transition toTenofovir/Lamivudine/Dolutegravir (TLD)}

Dolutegravir (DTG) is an integrase inhibitor (INSTI) that was first approved by the US FDA in 2013. It has shown clinical superiority in comparison to Efavirenz (EFV) in multiple large phase III trials. Additional benefits of DTG include quicker viral suppression, a high genetic resistance barrier, a long half-life, and low-dosing requirements. Cost of DTG regimen is also competitive. It has less toxicity profile and a smaller pill size that is treatment adherence-friendly. Other programmatic considerations that were instrumental to this transition include; product cost, lead time, number of manufacturers offering the fixed dose formulation and availability of other options for patients who may develop toxicity to DTG. Malawi processed an order for 6 million doses of TLD in January 2018. The cost per patient per annum based on Global Fund Pooled Procurement Mechanism (PPM) pricing as at January 2018 was USD75 and additional price reductions are expected in the near future.

\section{Implementation of Transition}

Malawi is planning for a national scale transition to the DTG based regimen (TLD) from beginning of 2019. The timing will allow for implementation of key transition activities over a period of 12 months. These include; finalization of 2018 care and treatment guidelines, procurement of over 6 million doses of TLD and distribution of the new product in time for the transition, capacity building activities, development of communication materials for providers, patient groups and Civil Society Organizations (CSOs) and stakeholder meetings amongst other critical activities.

Like all transitions, a few clinical components require critical thinking and hence the need for pilot implementation given that most experiences in Bostwana, Kenya and other countries have focused on experiences in 
treatment of naïve patients. Important variables such as cost, laboratory capacity, and confirmation of Viral Load suppression on the current regimen will be evaluated in the process of switching patients to the new regimen.

There is no sufficient information on the prevalence of Nucleoside Reverse Transcriptase Inhibitors (NRTI) drugs resistance mutations among the approximately $10 \%$ of patients who are found with viral load more than 1000 (VL>1000) in routine monitoring.

The uncertainty around pre-existing NRTI resistance necessitated a pilot implementation in the second half of
2018. This will enable $\mathrm{MoH}$ to avert the risk of "dolutegravir monotherapy" and preserve future treatment options. Other patient groups of particular interest are those classified as "new initiation" and "reinitiation". The MoH is working with partners to fast track the pilot implementation in a few sites to answer questions that are critical to the transition.

\section{Reference}

1. 2017 Spectrum HIV population estimates. 